\title{
Research on Personalized Commodity Recommendation Method Based on the Fuzzy Semantics \\ Jingjing Jiang ${ }^{1, a^{*}}$, Sheng Guan ${ }^{1, b^{*}}, \mathrm{Yao} \mathrm{Li}^{1, \mathrm{c}}$ and Xiangwei $\mathrm{Mu}^{2, \mathrm{~d}}$ \\ ${ }^{1}$ Dalian Neusoft University of Information, 8 Software Park Road, Dalian 110623, Liaoning, China \\ 2Dalian Maritime University, 1 Linghai Road, Dalian 116026, Liaoning, China \\ ajiangjingjing@neusoft.edu.cn, b guansheng@neusoft.edu.cn, ${ }^{c}$ liyao@neusoft.edu.cn, \\ xiangwei.mu@gmail.com \\ * The Corresponding Author
}

Keywords: Fuzzy semantics; Personalized recommendation; E-commerce; FALC syntax; Interest degree

\begin{abstract}
In order to meet the need of semantics recommendation to personalized commodity in e-commerce, the model based on fuzzy semantics personalized recommendation system is built to describe the integrated user's interest feature and commodity information. The user's interest degree of commodity and the correlation degree of commodity and interest are described using FALC syntax, and the related algorithm is used to complete user's interest data-mining. The experiment shows that the personalized recommendation method and model based on fuzzy semantics can make the e-commerce system to describe fuzzy commodity concept and recommend more appropriate goods to users.
\end{abstract}

\section{Introduction}

With the vigorous development of computing network, Internet users and information providers are facing the embarrassing situation of "information starvation and information overload" due to the rapid growth of network information resources[1].Traditional recommendation system can be divided into rule-based recommendation method, collaborative filtering recommendation method[2], content-based recommendation method and network structure-based recommendation algorithm, and it faces the problems of cold start and data sparsity[3], which seriously affect the accuracy and timeliness of the recommended results.

In this paper, a personalized recommendation system model based on fuzzy semantics is established according to the research on the organization and management method and system model of personalized recommendation service in the field of electronic commerce to solve the personalized recommendation service problem in the semantic environment. It ensures that users can efficiently utilize and absorb commodity information resources in e-commerce activities, so that users' personalized information needs based on semantics can be satisfied.

\section{Fuzzy Description Logic}

AL (Attributive Language) is the most basic one of many multilanguage languages that are included in describe logic, but can only define atomic negative[4]. ALC (Attribute Language Complement) has improved the AL language on the basis of which the constructor is extended to express negation of any concept. Constructor includes disjunction $(\sqcup)$,conjunction $(\Pi)$,negation $(\neg)$, value limit $(\forall)$ and existence $\operatorname{limit}(\exists)$.But for uncertain knowledge reasoning and expression, ordinary description logic cannot be expressed accurately. In order to solve this problem effectively, Straccia proposed FALC (fuzzy extension logic of ALC) to help ALC to describe the fuzzy concept[5].

Definition 1:FALC fuzzy description logic 
FALC defines $\mathrm{C}|\mathrm{D}| \mathrm{C} \sqcup \mathrm{D}|\mathrm{C} \sqcap \mathrm{D}| \neg \mathrm{C}|\exists \mathrm{R} . \mathrm{C}| \forall \mathrm{R} . \mathrm{C}$ fuzzy concepts as $\mathrm{I}=\left(\Delta^{\mathrm{I}} \cdot{ }^{\mathrm{I}}\right)$. Among them, $\cdot{ }^{\mathrm{I}}$ expresses the mapping from the fuzzy concept to membership grade value $R^{\mathrm{I}}=\Delta^{\mathrm{I}} \times \Delta^{\mathrm{I}} \rightarrow[0,1], C^{\mathrm{I}}=\Delta^{\mathrm{I}}$ $\rightarrow[0,1]$, and satisfy the following equation:

$\wedge^{\mathrm{I}}(\mathrm{d})=0, \mathrm{~T}^{\mathrm{I}}(\mathrm{d})=1,(\mathrm{C} \sqcup \mathrm{D})^{\mathrm{I}}(\mathrm{d})=\max \left\{\mathrm{C}^{\mathrm{I}}(\mathrm{d}), \mathrm{D}^{\mathrm{I}}(\mathrm{d})\right\},(\mathrm{C} \sqcap \mathrm{D})^{\mathrm{I}}(\mathrm{d})=\min \left\{\mathrm{C}^{\mathrm{I}}(\mathrm{d}), \mathrm{D}^{\mathrm{I}}(\mathrm{d})\right\}, \neg \mathrm{C}^{\mathrm{I}}(\mathrm{d})=1-\mathrm{C}^{\mathrm{I}}(\mathrm{d})$, $(\exists R . C)^{\mathrm{I}}(\mathrm{d})=\sup _{\mathrm{d}^{\prime} \in \Delta \mathrm{I}} \min \left\{\mathrm{R}^{\mathrm{I}}\left(\mathrm{d}, \mathrm{d}^{\prime}\right), \mathrm{C}^{\mathrm{I}}(\mathrm{d})\right\},(\forall \mathrm{R} . \mathrm{C})^{\mathrm{I}}(\mathrm{d})=\inf _{\mathrm{d}^{\prime} \in \Delta \mathrm{I}} \max \left\{1-\mathrm{R}^{\mathrm{I}}\left(\mathrm{d}, \mathrm{d}^{\prime}\right), \mathrm{C}^{\mathrm{I}}(\mathrm{d})\right\}$

This paper uses the introduction of a FALC (D) with a specific domain to implement the recommended model[6].

Definition 2:specific domain fuzzy description logic FALC(D)

$$
\begin{array}{r}
\mathrm{C} \rightarrow \mathrm{T}|\wedge| \mathrm{A}\left|\mathrm{C}_{1} \sqcap \mathrm{C}_{2}\right| \mathrm{C}_{1} \sqcup \mathrm{C}_{2}|\neg \mathrm{C}| \forall \mathrm{R} . \mathrm{C}|\exists \mathrm{R} . \mathrm{C}| \forall \mathrm{T} . \mathrm{D}|\exists \mathrm{T} . \mathrm{D}| \mathrm{m}(\mathrm{C}), \mathrm{D} \rightarrow \mathrm{u}\left|\neg \mathrm{u}, \mathrm{u} \rightarrow \operatorname{trz}\left(\mathrm{a}, \mathrm{b}, \mathrm{c}, \mathrm{d},\left[\mathrm{k}_{1}, \mathrm{k}_{2}\right]\right)\right| \\
\operatorname{tri}\left(\mathrm{a}, \mathrm{b}, \mathrm{c},\left[\mathrm{k}_{1}, \mathrm{k}_{2}\right]\right)\left|\operatorname{ls}\left(\mathrm{a}, \mathrm{b},\left[\mathrm{k}_{1}, \mathrm{k}_{2}\right]\right)\right| \operatorname{rs}\left(\mathrm{a}, \mathrm{b},\left[\mathrm{k}_{1}, \mathrm{k}_{2}\right]\right) \mid \operatorname{cr}\left(\mathrm{a}, \mathrm{b},\left[\mathrm{k}_{1}, \mathrm{k}_{2}\right]\right), \mathrm{m} \rightarrow \operatorname{lm}(\mathrm{a}, \mathrm{b}, \mathrm{c}, \mathrm{d})
\end{array}
$$

The $\mathrm{D}$ is an element of fuzzy predicate, such as: love, $\leq \mathrm{x} . \mathrm{M}$ is a fuzzy modifier, such as very, very much, general, and so on. $\operatorname{lm}(\mathrm{a}, \mathrm{b}, \mathrm{c}, \mathrm{d})$ and trz $(\mathrm{a}, \mathrm{b}, \mathrm{c}, \mathrm{d},[\mathrm{k} 1, \mathrm{k} 2])|\operatorname{tri}(\mathrm{a}, \mathrm{b}, \mathrm{c},[\mathrm{k} 1, \mathrm{k} 2])| \mathrm{ls}(\mathrm{a}, \mathrm{b},[\mathrm{k} 1$, $\mathrm{k} 2])|\mathrm{rs}(\mathrm{a}, \mathrm{b},[\mathrm{k} 1, \mathrm{k} 2])| \mathrm{cr}(\mathrm{a}, \mathrm{b},[\mathrm{k} 1, \mathrm{k} 2])$ are different membership function models. They are as shown in Fig. 1.

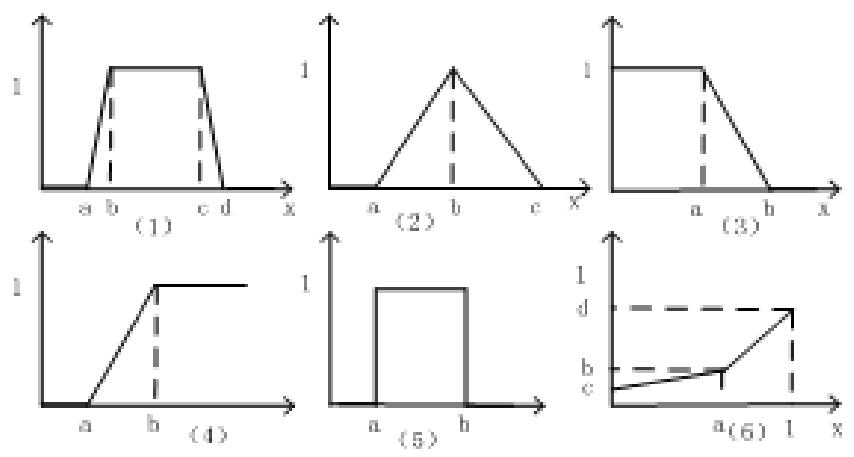

Figure 1. Finite Fuzzy description logic membership function

\section{Construction of Personalized Semantic Recommendation System}

Semantic Description Model of Fuzzy Semantic Recommendation System. The semantic description model of the fuzzy semantic recommendation system is mainly used to describe the relationship between the user and the interest, the interest and the resource[7]. The definition of an interest semantic description model for the semantic recommendation system is as follows[8]:

Definition 3: Interest semantic description model SRS is a six tuple: $S R S=\langle U, I, R, P U I, P I R, F\rangle$

$\mathrm{U}$ is a user concept set, which is used to describe the type and feature attribute of the user.I is a set of interest concepts, such as office supplies, electronic digital, food and beverage, sports outdoor and so on. $\mathrm{R}$ is a concept set of online commodity resources, which is used to describe the characteristics of the goods.PUI is the semantic relationship between the concepts of U and I, PUI $\in$ $\mathrm{U} \times \mathrm{I}$, which can be represented as a two tuple pUI(u,i),pUI $\in \mathrm{PUI}, \mathrm{u} \in \mathrm{U}, \mathrm{i} \in \mathrm{I}$. Such as: hasInterest (a user, office supplies).PIR is the semantic relationship between $I$ and $R, P I R \in I \times R$, which can be represented as a two tuple $\operatorname{pIR}(r, i), p I R \in P I R, i \in I, r \in R$. Such as: correlate (a commodity, electronic digital). $\mathrm{F}$ is a set of background knowledge and related rules, such as the semantics of terms such as hot commodities.

Implementation of Fuzzy Semantic Recommendation System Based on FALC. In this paper, the semantic description model of interest is implemented based on FALC. The implementation of the model is mainly composed of five processes.

Create a user profile (User Profile, UP), which describes the semantic relationship $\mathrm{P}_{\mathrm{UI}}$ between user $\mathrm{U}$ and interest I in the form of FALC syntax.

Establish the Resourse Profile (RP), which describes the semantic relationship $\mathrm{P}_{\mathrm{IR}}$ between the resource $\mathrm{R}$ and interest I in the FALC syntax form. 
Interest Profile (IP), which describes the concepts in a certain domain in the form of the syntax of FALC.

Define fuzzy concept set $\mathrm{F}$ for background knowledge.

Establish the relationship between users and resources by defining the recommendation degree between users and resources. Set the calculation function of the degree of recommendation as $\prod^{+}\left(\mathrm{p}_{1}, \mathrm{p}_{2}\right)>0$, in which $\mathrm{p}_{1}, \mathrm{p}_{2} \in\left\{\geq_{\mathrm{d}}, \leq_{\mathrm{d}},=_{\mathrm{d}}\right\}$, and $\mathrm{d}$ is the value of characteristic domain. Obviously, the user is interested in a concept often implies that the user is intrested in its parent concept in some degree.

$\mathrm{P}_{\mathrm{UI}}$ rule: $\mathrm{i}_{1} \sqsubseteq \mathrm{I}, \quad \mathrm{i}_{2} \subseteq \mathrm{I}, \quad \mathrm{i}_{1} \sqsubseteq \quad \mathrm{i}_{2} \wedge \exists$ hasInterest.(i $\Pi \geq_{\mathrm{x}}($ interestLevel)$) \rightarrow \exists$ hasInterest. $\left(\mathrm{i}_{2} \Pi\right.$ $\geq_{\Phi(\mathrm{x})}($ interestLevel)). $\Phi(\mathrm{x})$ is the conversion function of the degree of interest,which will map the degree of interest in subconcepts to the degree of interest in the parent concept. For example,the concept of mobile goods is a sub concept of electronic digital goods.If a user's interest degree of mobile phone is 6 , according to the PUI rule, his interest degree in electronic digital goods is at least $\Phi(6)$.

$\mathrm{P}_{\mathrm{IR}}$ rule: $\mathrm{i}_{1} \sqsubseteq \mathrm{I}, \quad \mathrm{i}_{2} \subseteq \mathrm{I}, \quad \mathrm{i}_{1} \subseteq \mathrm{i}_{2} \wedge \exists$ correlate. $\left(\mathrm{i}_{1} \Pi \geq_{\mathrm{x}}\right.$ (correlatorLevel)) $\rightarrow \exists$ correlate. $\left(\mathrm{i}_{2} \Pi \geq\right.$ $\theta(\mathrm{x})($ correlatorLevel $)) . \theta(\mathrm{x})$ is the conversion function of correlation degree, which will map the correlation between the sub concept of interest and the resource to the correlation between the parent concept of interest and the resource. For example, stationery consumables are a kind of office supplies. If the association degree of a commodity and stationery consumables is 7 , the association degree of a commodity and office supplies resources is $\theta(7)$.

The calculation process of a resource and a user's recommendation degree is defined as doRecommendation(UP,RP), and the program is shown in Table 1.

Table 1 Recommendation degree algorithm

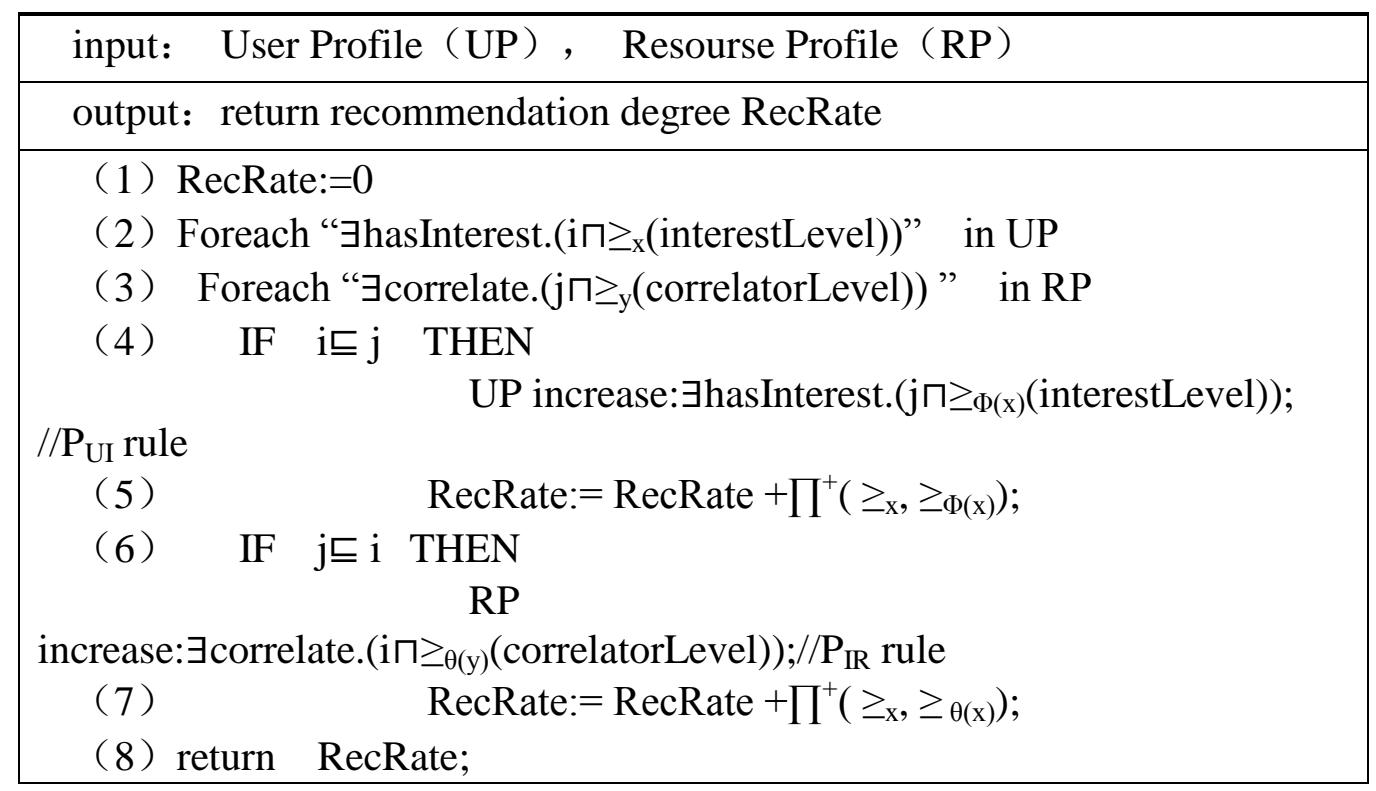

\section{Instance}

Instance Description. In e-commerce recommendation system, user profiles are built according to the basic information such as $\{\mathrm{u} 1, \mathrm{u} 2, \mathrm{u} 3\}$, browsing records and comment information of users.PUI is composed of all user profiles.Create a resource profile for each item in the system. PIR is composed of all resource profiles in the system. Some of the contents are as follows:

Complete system T:(user, resource, interest);

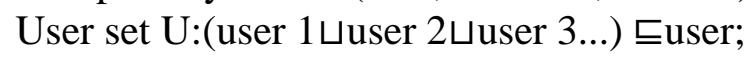

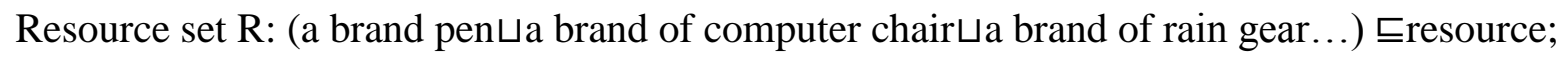


Interest concept set I:(home furnishingsபoffice suppliesபelectronic digital products)

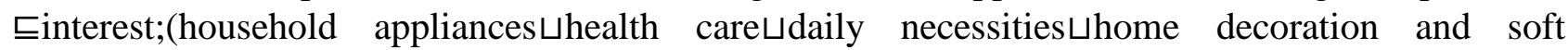

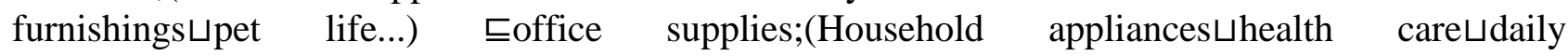

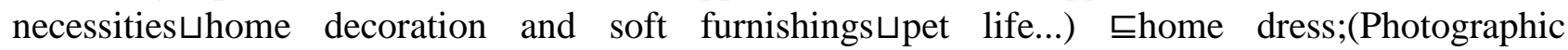
video $\sqcup$ audio-visual equipment $\sqcup$ storage devices $\sqcup$ network equipment $\sqcup$ mobile phones...) ㅌelectronic digital goods;

User interest description $\mathrm{P}_{\mathrm{UI}}$ :

User $1 \sqcap \exists$ hasInterest.(health care $\Pi \geq_{8}$ (interestLevel)) $\Pi \exists$ hasInterest.(photocopier equipment $\Pi \geq \geq_{6}($ interestLevel) $) \Pi \exists$ hasInterest.(network equipment $\Pi \geq_{6}($ interestLevel));

User $2 \sqcap \exists$ hasInterest.(health care $\Pi \geq 8$ (interestLevel)) $\Pi \exists$ hasInterest.(photography and vedio recording $\Pi \geq 8$ (interestLevel)) $\Pi \exists$ hasInterest.(home decoration $\Pi \geq 8$ (interestLevel)) $\Pi \exists$ hasInterest.(office consumables $\Pi \geq 8$ (interestLevel));

User $\quad 3 \sqcap \exists$ hasInterest.(pet $\quad$ life $\Pi \geq_{8}$ (interestLevel)) $\Pi \exists$ hasInterest.(daily necessities $\Pi \geq 6$ (interestLevel)) $\Pi \exists$ hasInterest.(photography and vedio recording $\Pi \geq_{8}($ interestLevel) $) \Pi \exists$ hasInterest.(network equipment $\Pi \geq_{8}($ interestLevel));

Resource interest Association description table $\mathrm{P}_{\mathrm{IR}}$ :

A brand pen $\Pi==_{\text {January }} 2017$ (release time) $\Pi={ }_{9}$ (browsing times) $\Pi={ }_{6}$ (praise times) $\Pi={ }_{1}$ (bad review times) $\Pi \exists$ correlate.(office supplies $\Pi \geq_{5}$ (correlatorLevel));

A brand computer chair $\Pi={ }_{\text {March }} 2017$ (release time) $\Pi={ }_{1}$ (browsing times) $\Pi \exists$ correlate.(home furnishing $\Pi \geq_{8}$ (correlatorLevel) $) \sqcap \exists$ correlate.(office supplies $\Pi \geq_{6}$ (correlatorLevel));

A brand rain gear $\Pi=$ July 2017 (release time) $\Pi==_{10}$ (browsing times) $\Pi==_{3}$ (praise times) $\sqcap \exists$ correlate.(home furnishing $\Pi \geq_{8}$ (correlatorLevel));

A brand intelligent switch $\Pi={ }_{\text {April }} 2017$ ((release time) $\Pi={ }_{8}$ (browsing times) $\Pi_{6}{ }_{6}$ (bad review times) $\Pi \exists$ correlate.(electronic digital $\Pi \geq_{4}$ (correlatorLevel) $\Pi \exists$ correlate.(home furnishing $\Pi \geq 6$ (correlatorLevel));

A brand computer $\Pi=$ April 2017 (release time) $\Pi==_{7}$ (browsing times) $\Pi==_{2}$ (praise times) $\Pi={ }_{3}$ (bad review times) $\Pi \exists$ correlate.(office supplies $\Pi \geq_{8}$ (correlatorLevel)) $\sqcap \exists$ correlate.(electronic digital $\Pi \geq \geq_{8}($ correlatorLevel));

A brand cartridge $\Pi=$ February 2017 (release time) $\Pi={ }_{3}$ (browsing times) $\Pi \exists$ correlate.(photocopier equipment $\Pi \geq_{8}($ correlatorLevel) $) \Pi \exists$ correlate.(office consumables $\Pi \geq_{6}($ correlatorLevel));

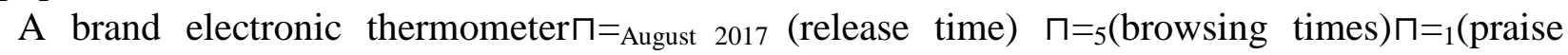
times) $\Pi==_{1}($ bad review times) $\Pi \exists$ correlate.(household electric appliances $\Pi \geq_{5}($ correlatorLevel $\left.)\right) \Pi \exists$ correlate.(health care $\Pi \geq_{6}($ correlatorLevel $\left.)\right)$;

A brand network storage $\Pi=$ September 2017 (release time) $\Pi \exists$ correlate.(storage device $\Pi \geq_{8}($ correlatorLevel) $) \Pi \exists$ correlate.(network equipment $\Pi \geq_{7}($ correlatorLevel));

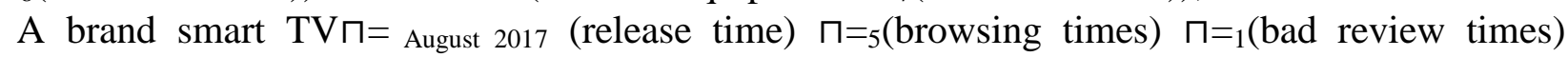
$\Pi \exists$ correlate.(audio and video equipment $\Pi \geq_{3}$ (correlatorLevel)) $\Pi \exists$ correlate.(household electric appliances $\Pi \geq_{8}($ correlatorLevel));

A brand router $\Pi=$ January 2017 (release time) $\Pi={ }_{11}$ (browsing times) $\Pi={ }_{6}$ (praise times) $\sqcap \exists$ correlate.(network equipment $\Pi \geq_{9}$ (correlatorLevel)) $\Pi \exists$ correlate.(office supplies $\Pi \geq_{5}$ (correlatorLevel));

Background knowledge F:

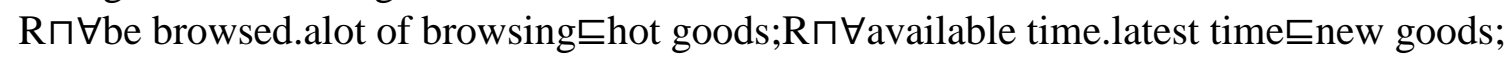

It is necessary to first define $\Phi(\mathrm{x}), \theta(\mathrm{x})$ in $\mathrm{P}_{\mathrm{UI}}$ rule and $\mathrm{P}_{\mathrm{IR}}$ rule before computing the recommended intensity of user and resources. Let $\mathrm{i}$ be a subconcept of $\mathrm{j}$, and $\mathrm{i} \sqsubseteq \mathrm{j}, \Phi(\mathrm{x})=\theta(\mathrm{x})=$ $(|\mathrm{i} / \mathrm{j}| \mathrm{j} \mid) \mathrm{x}$, in which $|\mathrm{i}|$ and $|\mathrm{j}|$ are the model of $\mathrm{i}$ and $\mathrm{j}$. Secondly, the positive recommendation intensity and negative recommendation intensity between the user and the resource are defined: $\Pi^{+}\left(\geq_{x}\right.$, $\left.\geq_{y}\right)=x \cdot y$

The definition of membership function is as follows:

HighinerestLevel $($ interestLevel $)=\mathrm{rs}(40,70,[0,100])$; 
CommonInerestLevel(interestLevel $)=\operatorname{trz}(30,40,60,70,[0,100])$;

lowInerestLevel (interestLevel) $=1 \mathrm{~s}(20,50,[0,100])$;

HighCorrelateLevel (correlateLevel $)=\operatorname{rs}(40,70,[0,100])$;

CommonCorrelateLevel(correlateLevel $)=\operatorname{trz}(30,40,60,70,[0,100])$;

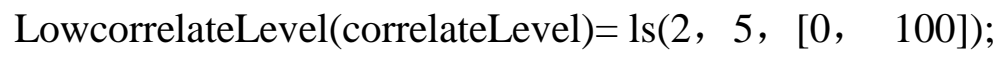

The membership function of the fuzzy features in IP: browse many times (Views) $=$ rs (Q3, Max, $[0, \max ])$; the latest time (current time - release time) $=1 \mathrm{~s}(3,6,[0,12])$, in which Q2, Q3 is two and three quantiles in four quartiles of the total number of resources, and max is the maximum number of times. The recommended results are shown in Table 2.

Table 2 List of recommended results

\begin{tabular}{|c|c|}
\hline user & ranking of recommended results (the first five) \\
\hline user1 & $\begin{array}{c}\text { (a brand router })>(\text { a brand cartridge })=(\text { a brand electronic thermometer })>(\text { a brand } \\
\text { computer chair })>(\text { a brand computer })\end{array}$ \\
\hline user2 & $\begin{array}{c}\text { (a brand cartridge })>(\text { a brand electronic thermometer })>(\text { a brand computer chair })> \\
(a \text { brand intelligent switch })>(\text { a brand computer })\end{array}$ \\
\hline user3 & $\begin{array}{c}\text { (a brand router })>(\text { a brand network storage })>(\text { a brand computer })=(\text { a brand rain } \\
\text { gear })>(\text { a brand computer chair })\end{array}$ \\
\hline
\end{tabular}

Analysis of Recommended Results. The recommended results are extensible. It can be seen from the recommended results that some of the selected resources that should be recommended to the user can not be recommended to the user in a keyword based approach. And the recommended results increase the ability to describe the fuzzy concept.

\section{Conclusion}

This paper mainly studies the fuzzy semantic personalized recommendation method based on FLAC syntax, and proposes a commodity recommendation model and algorithm to deal with fuzzy semantic information. The experimental results show that the fuzzy semantic personalized recommendation system model can improve the recommended diversity problems in the following traditional models, lack of semantic information and cold start problem, and enhance the ability of fuzzy concept description.

How to automatically extract semantic descriptions related with user interests and commodity interest from structured and semi-structured user behavior information and commodity information in e-commerce will be the future research direction.

\section{Reference}

[1] H.B. Wang:Preference Recommendation for Personalized Search, Vol. 100(2016) No.15, p.124.

[2] Z.B. Wu,Y. Chen and T.Y Li:2014 International Conference on Information Science, Electronics and Electrical Engineering(Sapporo,Japan,April 26-28,2014),Vol.13, p.201.

[3] Z. Zhang, H. Lin and K. Liu:A Hybrid Fuzzy-Based Personalized Recommender System for Telecom Products/Services, Vol. 235(2013) No.20, p.117.

[4] S.H. Cai,J.M. Jun and Z.L. Yang: Journal of Operations Management,Vol.28(2014) No.3, p.257.

[5] C.M. Karat, J.O. Blom and J. Karat: Designing Personalized User Experiences in E-Commerce(Springer, Netherlands 2014),p.1040

[6] T. Nakano,K. Honda and S.Ubukata: 2016 8th International Conference on Soft Computing and Intelligent Systems(Sapporo,Japan,August 25-28,2016),Vol.2,p.96. 
[7] G. Castellano, A.M. Fanelli and M.A. Torsello: Applied Soft Computing, Vol. 11(2014) No.1, p.793.

[8] D. Anand and B. S. Mampilli: Expert Systems with Applications, Vol. 41(2014) No.5, p.2424.

[9] K. AlmohammadiEmail,H. Hagras and B. Yao:Soft Computing,Vol. 21(2017) No.4, p.965.

[10]Q. Zhang,D.S. Wu,G.Q. Zhang and J. Lu:2016 IEEE International Conference on Fuzzy Systems(Vancouver,Canada,July 24-29,2016),Vol.20,p.257. 